\title{
Traduire le mouvement du sens: Germen et Gestalten en sémantique textuelle des formes
}

\author{
France DUPUIS \\ $L L L$ Université d'Orléans \\ France
}

Résumé: Cette étude a pour but de convaincre les professionnels de la traduction qu'avant même de commencer à traduire, il faut se familiariser avec une théorie du sens. C'est seulement alors qu'il devient possible d'obtenir une lecture et une interprétation sémantique efficiente du texte-source.

Nous proposons une sémantique textuelle élaborée à partir de travaux initiés en sémantique lexicale ainsi qu'une analyse des problématiques de la signification développées en philosophie des formes symboliques, en phénoménologie et dans d'autres travaux, notamment ceux de E. Coseriu. Afin d'illustrer nos propos théoriques nous traitons un exemple d'analyse sémantique à partir du poème Chanson noire de $\mathrm{L}$. Aragon.

Mots-clés: Pré-requis du traducteur, théorie du sens, interprétation sémantique, sémantique textuelle des formes, théorie des formes sémantiques, canevas sémantique, problématique de la signification, parcours sémantique de l'énonciatif, phénoménologie, Aragon, Chanson noire.

\begin{abstract}
This study aims at convincing translation specialists that prior to the very act of translating, it is essential to become familiar with a Theory of Meaning. Only then will it become possible to bring about a truly efficient reading and a semantic interpretation of the original text. Based on works initiated in Lexical Semantics, an original Text Semantics approach is suggested, as well as an analysis of the questions of Meaning carried out in the Philosophy of Symbolic Forms, in Phenomenology and in other works, notably those of E. Coseriu. To illustrate this theoretical framework, we offer in the semantic analysis of Chanson noire, a poem by Louis Aragon.
\end{abstract}

Key words: Translator's prerequisites, theory of meaning, semantic interpretation, text semantics, theory of semantic forms, semantic framewok, semantic canvas, issues in meaning approach, semantic scanning of the utterer-centered field, phenomenology, Aragon, Chanson noire.

Comment traduire le mouvement du sens, la germination du sens ? Comment saisir ce processus et la potentialité signifiante qui est un préalable aux choix des unités lexicales que le traducteur va convoquer dans la langue-cible et dont il va repérer les traces d'un vouloir-dire dans la langue-source ? C'est à ces questions que notre 
analyse tente de répondre au travers d'une approche sémantique des formes.

Notre travail vise à proposer des pistes de réflexion aux professionnels de la traduction en les invitant à découvrir une sémantique textuelle des formes comme une possible lecture et un canevas d'interprétation du texte-source en amont du travail du traduire (Dupuis, 2013, 279-285). Sur la question d'une problématique du sens en lien avec le traduire, nous citons à ce sujet J-R Ladmiral: « comment dire le sens ? dès lors qu'il est « désincarné » de l'énoncé qui en était porteur. Il s'agira de verbaliser et de conceptualiser le sens, au moment où l'on est dans cet entre-deux de la traduction où il n'est plus gagé ni sur les signifiants du texte-source (To), et pas encore sur ceux du texte-cible (Tt) » (Ladmiral, 2006, 115).

L'analyse s'inscrit ainsi nécessairement dans une bipartition théorie et pratique. Son but ultime est de préparer le traducteur à la nécessité ultérieure de dégager une méthode d'analyse et de saisie du mouvement du sens et du foisonnement des potentialités. Nous ouvrirons la possibilité d'un débat à venir sur notre démarche d'une sémantique textuelle phénoménologiquement inspirée aux traducteurs lorsque ces derniers s'interrogent sur la complexité du vouloir-dire

Ce travail de recherche sur une sémantique des formes repose sur une analyse linguistique, sémantique et sémiotique qui s'inscrit dans la continuité des travaux entrepris par Cadiot et Visetti, en sémantique lexicale (Cadiot et Visetti, 2001). Notre intérêt porte sur le projet de développement d'une sémantique textuelle s'inspirant des travaux linguistiques précédemment cités, tout en revisitant les théories gestaltistes - dont l'école de Berlin (Khöler, Koffka), les travaux du philosophe Cassirer sur les formes symboliques - ainsi que les travaux d'inspiration phénoménologique (Husserl, Merleau-Ponty, Bachelard, Henry pour ne citer que les principaux auteurs qui viennent alimenter notre réflexion mais que nous ne développerons pas ici). Notre travail repose, d'autre part, sur l'étude des travaux de linguistes dont, entre autres, Coseriu, que nous citerons dans une optique «phénoménologique » de l'étude du langage.

Notons que le passage du monde des phénomènes à un monde signifiant s'accompagne d'un profilage de valeurs au niveau du langage dans un parcours motivationnel et thématique de la parole. Au niveau $\mathrm{du}$ discours et du texte, le profilage syntagmatique et les parcours motivationnels et thématiques constituent des strates de sens entremêlées que l'on peut, néanmoins, isoler au niveau du lexème, de la phrase ou du texte. En outre, dans notre approche, nous veillons à ce que les outils d'analyse textuelle ne bloquent pas l'interprétation de contenus sémantiques sous-jacents que nous appelons "germes de sens » ou Germen et dont on peut retrouver les traces dans les profilages des valeurs au niveau syntagmatique.

Dans un premier temps, afin d'expliciter notre travail, nous poserons les bases théoriques d'une sémantique textuelle des formes en 
lien avec les théories des formes sémantiques et des problématiques propres au traduire.

Dans un second temps, nous proposons l'exemple d'une analyse sémantique textuelle des formes, celle du poème Chanson noire d'Aragon extrait du recueil Elsa.

\section{Sémantique textuelle des formes et «traduire »}

\subsection{L'apport de la philosophie des formes symboliques}

La philosophie des formes symboliques apporte un éclairage particulier à la problématique de la signification quant au rapport entretenu avec le langage dans l'accès au monde. Le langage permet un accès au monde et une donation des objets. Cassirer met notamment en avant le mythe, le rite et plus généralement le langage dans la sémiotisation du monde. Nous pouvons également évoquer les archétypes, entre mythe et onirisme qui alimentent l'inconscient individuel et collectif. Ces "matériaux» se retrouvent de façon indistincte dans le langage et dans les langues sous forme d'esquisses perceptuelles. Citons à ce propos l'exemple proposé par Cassirer, exemple emprunté à Humboldt, au sujet du mot lune en grec puis traduit en latin, qui souligne d'une part cet aspect de la langue, à savoir ces traces perceptuelles, floues qui viennent marquer le langage, et d'autre part la difficulté qui en découle dans la traduction. Nous citons Cassirer: " Comme Humboldt l'a fait remarquer, les termes grec et latin désignant la lune, bien qu'ils se rapportent au même objet, n'expriment pas la même intention ou le même concept. Le terme grec (men) désigne la fonction de la lune qui est de mesurer le temps, le terme latin (luna, lucna) désigne la luminosité ou l'éclat de la lune. Nous avons fixé notre attention sur deux caractères très différents de l'objet que nous avons nettement isolés. » (Cassirer, 1975, 192-193).

Rappelons, brièvement ici, que l'objectif principal des théories des formes, dont nous nous inspirons, vise à dégager un principe actif de la «forme» tel qu'il puisse être utilisé dans de nombreuses approches scientifiques. La "forme »- dont la notion est développée par Cassirer dans sa philosophie du langage et empruntée par ce dernier à Kant - est appréhendée comme étant organisée ; elle est à la fois dynamique, changeante et par conséquent instable. Ajoutons que la " forme » est instituée d'une façon collective. De ce fait, elle apparaît dans toute activité sémiotique et permet une forme d'accès au monde via le langage. Le passage de la substance - considérée comme sensible, pressentie, intuitive - à la forme - davantage intelligible et signifiante - marque le processus de formation des valeurs.

Cette instabilité de la forme en langue-source doit être d'abord repérée par le traducteur, avant que ce dernier ne décide, éventuellement de choisir dans le texte-cible une forme qui pourrait faire écho à cette instabilité de départ. Il est bien entendu hors de question, pour ce faire, de renier l'objectif résolument cibliste, tel que 
l'a défini à de nombreuses reprises Jean-René Ladmiral, et plus particulièrement dans son dernier ouvrage Sourcier ou cibliste.

\subsection{Une approche phénoménologique}

Au départ, disons que le monde qui fait sens pour le sujet est un monde de phénomènes qui subit la pression des transformations en monde de signes dont les valeurs marquent le langage en un profilage permanent. Notons deux opérations élémentaires en phénoménologie: la visée et la saisie. La signification émerge de la perception dans une activité sémiotique mettant en exergue un système de valeurs. Autrement dit encore, le champ de la présence est traversé par le flux des Gestalten, des figures qui fluctuent, apparaissent et disparaissent. L'ensemble du champ est animé par la visée et la saisie, et traversé par des flux, des figures, des formes mais aussi des plis, des creux et des vides qui constituent la texture, la densité et le plein des significations et du sens.

L'un des postulats de base développé dans les travaux en phénoménologie, en particulier ceux d'inspiration merleau-ponthienne, est de mettre en avant une analogie entre activité de langage et activité perceptive. Autrement dit, il s'agit d'observer de quelle manière la langue participe dans la création de l'expérience par une sorte de reprise qualifiée de la phase de perception. Précisons que dans ce cadre la perception est bien plus qu'un simple enregistrement de données sensorielles, c'est en effet déjà l'expérimentation d'impressions esthétiques, l'esquisse de jugements de valeur et la préparation à l'action. Notons que dans notre approche, l'activité du langage est envisagée au sein de l'activité générale du sujet.

\subsection{Une sémantique textuelle des formes}

Notre objet d'étude, le texte, à traduire certes mais d'abord à reconnaître comme tel et à identifier, est appréhendé en tant qu'univers de valeurs dont les significations parviennent au lecteur dans la saisie de la singularité du jeu des formes linguistiques, une singularité qui marque le discours et permet une autre saisie - celle que le texte met en scène - partielle d'un réel possible (ou ne serait-ce que de ses interstices) circonscrit dans le champ virtuel du narratif. Le processus métaphorique et métonymique, central et crucial dans les formes textuelles, joue dans les rebonds et tensions du langage et de la langue, en profilage de ses figures et thématiques, tirant de l'ornière des motivations enfouies. Le texte répond à un dire et à ses principes d'association et d'évocation, celui lié aux expériences des pulsions scopiques $^{1}$ et des signifiances du désir du sujet qui commandent à la

\footnotetext{
${ }^{1}$ Nous faisons référence à Lacan dans « Le stade du miroir comme formateur de la fonction du Je » in Ecrits, 1966 (97). Lacan donne la primauté à l'image de l'autre comme formatrice du moi et à l'objet « regard ». On parle de « captation », de « libido » ou encore désir du voir. Cependant, comme le remarque Lacan, le stade du miroir conduit également « au leurre de l'identification spatiale » et «machine les fantasmes
} 
parole en bouffées narratives. Le traducteur ne saurait ignorer cette " réponse à un dire » ou alors seulement aux dépens de lacunes graves dans la restitution du vouloir-dire du texte-source dans le texte-cible.

La sémantique textuelle porte son intérêt sur ce que le texte donne à voir, sur les champs thématiques et motivationnels qu'il ouvre dans le narratif, et sur l'objet-texte dans ses constructions syntaxiques, syntagmatiques au travers des profilages. Autrement dit encore, le texte comme objet d'étude nécessite une étude sémantique textuelle qui l'appréhende dans sa formation et sa forme, et également dans l'émergence des formes sémantiques qui le traversent et constituent la sémantique du texte. Il y a à montrer la phénoménologie du texte et de la phrase: pointer sur l'objet-texte dans son champ de réalisation et pointer sur le rapport à l'objet qui peut être celui du lecteur au sens large ou plus spécifiquement celui du lecteur-traducteur.

Les paroles de l'écrivain, telles celles d'Hélène Cixous soulignent bien la phénoménologie du mot qui renvoie à une perception des choses qui semble totale alors qu'elle n'est jamais que fragmentée:

« Les mots surgissent où je n'attendais rien, ils crèvent la surface des choses simples comme des abcès, ils divisent ce qui m'avait toujours paru fait d'une seule pièce » (Cixous, 1986, 35).

Nous remarquons à quel point la langue et les opérations prédicatives permettent à tout locuteur l'élaboration de scénarios d'objectivation (fictive) à partir d'une réalité, ou plus précisément de la (re)construction de la réalité. Autrement dit encore, le langage et la langue sont les vecteurs favorisant la saisie du monde et la sémiose. Le monde, considéré dès le départ comme sémiotique, fait sens pour le sujet qui l'habite. Une forme n'est pas une forme figée, on parle de forme d'accès « à... » des rapports intérieurs et extérieurs à la parole et au discours. Une forme c'est également un ensemble de rapports de forces en œuvre, de tensions significatives dans la mesure où elles participent de l'actualisation de l'événementiel dans le discours et par le discours et de l'actualisation du figural dans le discours.

Notons que nous utilisons la notion de figure ou Gestalten car elle comporte cette idée de partialité comme accès à un tout. Nous tenons à préciser que l'on utilise l'expression "sens figural ", c'est-àdire un sens attaché à des figures, et non l'expression "sens figuré » pour bien se démarquer de l'étude du sens qui oppose "sens propre » au « sens figuré ». Il n'y a pas, selon nous, un sens « fort » qui serait

qui se succèdent d'une image morcelée du corps à une forme que nous appelons orthopédique de sa totalité et à l'armure enfin assumée d'une identité aliénante qui va marquer de sa structure rigide tout son développement mental » (97). Un corps morcelé que l'on retrouve souvent dans les rêves et qui peut expliquer, enfin émettonsnous l'hypothèse ici, la métonymie première du langage que l'on retrouve en langue et qui sert au sujet à appréhender une " réalité » qui se donne à lui. Rappelons qu'il n'y a de totalité du corps qu'imaginaire. 
premier et donc un sens propre sur un sens dit « figuré » pour parler du sens métaphorique. Le sens est toujours et d'emblée inscrit dans une dynamique potentiellement métaphorique et figurale et ouvre ainsi le champ possible de la parole et du discours.

Â ce sujet, citons L. Jenny pour qui « le figural témoigne de la capacité d'ouverture du discours. Il est pour une langue l'indispensable moyen de maintenir ouvert le champ de la désignation, c'est-à-dire d'accueillir la nouveauté d'un événement du monde, de rendre compte de son indescriptible non par une réduction aux significations admises, mais par un réaménagement du système linguistique » $(2009,135)$.

Sur le plan de la sémantique, nous nous distinguons, en effet, des sémantiques dites "représentationnelles - symboliques » qui considèrent que le mot présente des significations privilégiées. Ainsi en est-il de ce sens dit « littéral » tel qu'il serait partagé par l'ensemble de la communauté des locuteurs et que l'on trouve répertorié dans les dictionnaires de langue (par exemple: Trésor de la langue française informatisé, Le Grand Robert de la langue française).

La signification lexicale est alors basée sur la représentation des objets abstraits et concrets à partir de l'expérience. Le mot dans cette conception est dans un rapport symbolique à son référent c'est-à-dire à l'objet du monde auquel il réfère, et les propriétés de l'objet désigné sont aussi celles du nom qui lui correspond. Ce type de sémantique développe une approche du monde comme monde des objets, c'est-àdire un monde fixe. Cependant, on ne saurait passer sous silence la confusion souvent faite entre désignation et signification, et entre signification et "référence / réalité ", qui est un problème lié comme le rappelle, entre autres, Coseriu $(2001,24)$, au fait que le langage a longtemps été considéré comme domaine particulier de ce que l'on peut nommer «l'univers des signes ». Or, la question linguistique de la référence à proprement parler est à considérer, selon nous, comme une strate de sens parmi d'autres. Le traducteur, lui aussi, est confronté, en amont (langue-source) et en aval (langue-cible) à cette problématique.

Coseriu avance, en outre, que l'emploi du terme « signe » «peut facilement donner l'impression qu'il $y$ a un signandum prélinguistique » ce qui peut mener «à concevoir le langage comme un simple système de désignation - une nomenclature - pour des choses données d'avance, c'est-à-dire antérieures à la création des signes ». Ainsi, Coseriu poursuit au sujet du signe matériel, qu'il "sert tout d'abord à délimiter et rendre identifiables des significations, et ce n'est qu'en second lieu qu'il peut, en tant qu'associé à un signifié, être employé pour la désignation des choses extralinguistiques ». Ainsi, « la désignation découle de la signification » $(2001,24)$.

Notre sémantique des formes est de type indiciel dans le sens où il s'agit de développer une sémantique d'accès (Dupuis, 2011, 67). Dans cette approche, les objets ou référents n'existent pas indépendamment de leur saisie linguistique, et le monde servant de base d'étude n'est plus un monde fixe d'objets mais un monde mouvant de phénomènes, 
d'esquisses et de projets d'action (Dupuis, Actes $d u$ colloque Programme perception sémiotique et socialité. "Le thème perceptif en linguistique » mars 2010, à paraître), c'est-à-dire un monde d'expériences « pour » le sujet dans son rapport au monde. L'objet n'est pas figé, il est souvent dépassé et requalifié dans un cadre qui est celui de l'ouverture et de la co-présence. Le mot est vecteur de valeurs en tant qu'il est vécu phénoménologiquement; des valeurs qui construisent des modalités et potentialités d'action (Dupuis, 2011, 65).

\subsection{Principes métaphoriques et métonymiques au cœur de la langue}

Les discours ne sont pas des formes figées, limitées car le principe métaphorique du langage (que nous pouvons comprendre au sens large, c'est-à-dire incluant le langage de l'inconscient) est un ferment constitutif du langage. En effet, la métaphorisation, les opérations de métonymisation, la figuralité, permettent au sujet, aux actants dans le texte, d'investir les formes discursives, leur mouvement, dans l'apparaitre phénoménologique de l'étant-au-monde-et-aulangage pour reprendre l'expression merleau-ponthienne revisitée par Cadiot et Visetti (2001).

L'objet est l'expression des champs de l'évocation par le jeu de la figuralité. Une figuralité qui peut être analysée d'une façon synthétique par la sémantique textuelle des formes dans le jeu des profilages syntaxiques, dans les motivations ou Germen de sens qui fermentent le discours et par les thématisations en cours.

Notre approche souligne la place prépondérante des principes et processus métaphoriques, figuratifs, métonymiques visant à l'expression et la re-construction de valeurs significatives à partir d'un fond constitué de qualia sensibles qui vont se manifester dans l'émergence du distinctif, et d'impression fugaces, floues à la lisière de l'inconscient ou susceptibles d'émerger au détour d'une figure de l'inconscient.

Le traducteur croyant pouvoir faire l'économie de cette dernière problématique le ferait, une fois encore, aux dépens d'une restitution, certes fugace et labile dans son essence, du vouloir-dire d'origine. En effet, sans en faire bien entendu un pré-requis pour le traducteur, a fortiori pour le traducteur cibliste que nous avons vocation à être, on ne peut ignorer que le "signifiant » lacanien va dans ce sens, et plus généralement la fonction des rêves et de l'inconscient en psychanalyse dont l'analyse tend à montrer que l'inconscient ignore la distinction entre sens figurés et sens propres; la métaphore et la métonymie constituant l'essentiel du langage de l'inconscient. D'ailleurs, l'enfant, surtout le petit enfant, présente-t-il naturellement un langage " fleuri », facilement métaphorique, proche de ses instincts et désirs, tout droit issus de son inconscient. L'institutionnalisation du langage de l'enfant selon les normes lexicographiques, ainsi que son développement participent d'une régression et d'une disparition progressif de l'usage 
spontané de la figuralité dans son expression. Le phénomène de la figuralité sera regagné dans un second temps du développement du langage chez l'enfant.

\subsection{Parcours sémantique de l'énonciatif: les notions de motif, profilage et thème}

Il n'y a pas de linéarité du sens, mais des plans et des parcours du sens (Dupuis, Lautel-Ribstein, 2013), des rapports (intérieurs et extérieurs à la parole et à la langue) à des valeurs significatives. Ce modèle repose, comme nous l'avons dit précédemment, sur une phénoménologie de la perception dans laquelle la perception est définie comme d'emblée une activité sémiotique: "percevoir est une activité sémiotique qui repose sur la saisie immédiate de qualités et d'horizons [...] percevoir est toujours esquisser un sens qui ne se déploie que dans un parcours, dans une activité de thématisation par laquelle se font et se défont les identités » (Cadiot, Visetti, 2001, 50).

Une dialectique fond/forme sous-tend le modèle: les figures se détachent sur un fond favorisant la saillie d'une partie du fond, une forme sur le fond. Á ce titre la forme vient servir l'expression du fond. Notons des processus d'inversion de circulations entre fonds et formes comme les phénomènes sémantiques de métonymisation, de synecdochie par exemple. Le « fond » est un espace si l'on peut dire qui est marqué par le continu dans la discontinuité: le «trou » dans la parole à l'endroit de l'Autre, sur un plan psychanalytique, car le sujet porte en lui la béance liée au manque et favorisant le désir né de l'absence de la Mère.

Trois strates de sens co-déterminent l'organisation et l'activité sémantique dans la phrase et le texte et participent de la saisie sémiotique du monde au travers du parcours énonciatif: la motivation, le profilage et la thématisation.

Notre objectif est de faire émerger les couches ou régimes de l'appréhension progressive de la forme. Ces « couches » ou régimes de sens sont dégagés à des fins "artificielles », c'est-à-dire qu'elles n'apparaissent comme telles dans la lexie, mais permettent d'élaborer une sémantique textuelle (Dupuis, 2011 et Dupuis, Lautel-Ribstein, 2013).

$\mathrm{Au}$ niveau textuel, thématiques et motifs constituent le fond, deux des strates de sens qui alimentent l'activité sémantique, tandis que les formes linguistiques se profilent sur le plan syntagmatique.

Nous proposons dans l'élaboration d'une sémantique textuelle des formes un « schéma » de la scénographie qu'il s'agit d'identifier:

a) Un champ thématique de la présence: un texte comporte des acteurs ou actants qu'il s'agit d'identifier

b) Une dimension actantielle / intentionnelle

c) Une dimension temporelle

d) Un matériau de prédication 
e) La figuralité et plus généralement les processus de métaphorisation et métonymisation

f) Des motifs ou Germen de sens

g) Des profilages syntaxiques

h) Des thématisations en cours

Au niveau du motif, motivation ou Germen de sens notons:

1. Une dynamique de forces

2. Une dimension praxéologique: il s'agit d'un Germen de sens « actif », potentiel, virtuel mais qui favorise l'actualisation de ce matériau virtuel.

3. Une dimension axiologique: avec un halo de valeurs significatives

4. Une dimension esthésique: avec l'ouverture de la parole à la figuralité et aux principes de métaphorisation et de métonymisation qui favorise l'émergence de l'événementialité de la parole et de la vie consciente et inconsciente (si l'on se réfère aux travaux des psychanalyses freudiennes et lacaniennes).

Le figural est au centre de la strate de motivation. Notons qu'un même syntagme peut ouvrir sur plusieurs phases de sens. Ainsi le mot « nid »: nous le retrouvons dans un profilage syntagmatique comme « nid des oiseaux», dans une thématique comme celle du « nid d'amour » ou la thématique de la mère, et nous le trouvons encore dans le Germen ou motif de sens du " foyer ». La phase de motivation est une phase de mise en relation d'un motif linguistique, à des profilages et des thèmes.

Les motifs et thèmes servent de fond ou support à l'élaboration des opérations de profilage et de thématisation. La dialectique fond / forme qui caractérise la sémantique textuelle des formes repose sur l'articulation des strates de sens évoluant dans des glissements sémantiques entre fonds motivés et thématisés et formes profilées. Autrement dit, le profilage des formes se fait sur fond de motif(s) et thèmes tout en débouchant sur des thématiques toujours en cours de réalisation.

L'opération de thématisation repose, quant à elle, sur le thème perceptif, en tant que le thème est ce qui se dégage du champ thématique de la perception. Le thème perceptif s'il constitue un sens perçu est une esquisse qui n'est jamais achevée.

\section{Sémantique textuelle des formes du poème Chanson noire d'Aragon}

Le poème Chanson noire (Aragon, 2010), issu du recueil poétique Elsa, s'inscrit dans la continuité du discours amoureux de son auteur Louis Aragon adressé à « Elsa », prénom de l'épouse-écrivain 
Elsa Triolet qui devient expression et révélation de l'amour sublimé du langage poétique aragonien.

Nous allons explorer quelques pistes au travers d'une lecture et d'une étude du poème portant sur le rôle ontogonique de l'expression poétique dans ce texte, c'est-à-dire que l'expression n'est pas dans l'attente de réponse d'un autre, mais qu'elle fait exister ce qu'elle profère comme un acte achevé. Ainsi, le « je » poétique ne coïncide pas avec le « je » référentiel mais il pose une réalité - la réalité poétique comme existante par l'acte de parole poétique. Nous verrons que le sujet, dans ce poème contrairement à une construction poétique de base, ne se crée pas en même temps qu'il crée son objet. Notre hypothèse est que le langage poétique ici, un chant, est, chanté par une instance non-sujet, posé comme allocutaire du «je» poétique énonciatif. Notre intérêt portera sur l'expression " chanson noire », les différentes formes linguistiques et sémantiques qui lui sont associées ainsi que les thématiques.

\subsection{Situation de l'énonciation et étude du champ énonciatif}

Nous commençons par une étude du champ énonciatif et nous notons dans le texte les différents points suivants. La présence d'une première personne du singulier qui dénote la conscience énonciatrice d'un sujet sous la forme d'un pronom " je », qui est un embrayeur actant et qui renvoie donc au sujet de l'énonciation (le poète). Cette forme pronominale n'apparaît qu'à la quatrième strophe du poème: " Dire que je puis disparaître / Sans t'avoir tressé tous les joncs... ». On peut qualifier la présence de ce « je » comme faible puisqu'il n'y a que trois occurrences de la première personne du singulier dans le texte (aux $5^{\mathrm{e}}, 8^{\mathrm{e}}$ et $11^{\mathrm{e}}$ strophes).

Cela donne une suite séquentielle:

«Je puis disparaître » (4e)/ «Je ne regrette rien que» $\left(8^{\mathrm{e}}\right) /$ « Je t'aime » $\left(11^{\mathrm{e}}\right)$.

Notons ensuite les traces d'une deuxième personne du singulier, embrayeur qui renvoie au destinataire, avec la forme pronominale «tu » (pronom personnel), qui apparaît une première et seule fois dans le texte à la $5^{\mathrm{e}}$ strophe (« Sans faire flèche du matin / Flèche du trouble et de la fleur / De l'eau et de la douleur / Dont tu m'atteins »). Notons également une forme pronominale complément «t' » à la $4^{\mathrm{e}}$ strophe («Sans t'avoir tressé tous les joncs») et à la $11^{\mathrm{e}}$ strophe («Et pour crier-dieu que je t'aime »). Enfin, une forme pronominale possessive à la $4^{\mathrm{e}}$ strophe ( $\mathrm{A}$ ta fenêtre »), ainsi qu'à la $8^{\mathrm{e}}$ strophe («A ta mémoire »). Ce qui fait en tout cinq occurrences de la deuxième personne du singulier.

Nous obtenons la suite séquentielle suivante pour la deuxième personne du singulier: 
«Sans t’avoir tressé / Ta fenêtre / Tu m’atteins / Ta mémoire / Je t'aime »

Notons que les deux séquences contenant la forme possessive de la deuxième personne du singulier sont construites de la même façon: " A ta fenêtre / A ta mémoire ", c'est-à-dire un jeu sémantique et thématique sur la fenêtre comme mémoire.

Nous remarquons l'usage des pronoms possessifs: "mon", « ma », «mes » qui renvoient au rapport du possesseur qui est une première personne du singulier à l'objet possédé, avec treize occurrences dans le texte. L'énonciation débute par une suite de profilages syntaxiques contenant la forme du pronom possessif à la strophe 1: «Mon sombre amour... / Ma chanson.../ Mon quartier d'ombre... ».

Cette suite comporte également l'émergence de formes adjectivale «sombre» et nominale «ombre» en combinaison « sombre / ombre » qui renvoie à une motivation de la profondeur en mouvement. Autrement dit, "sombre » et «ombre » renvoient à la qualité du «noir», mais également à ce qui est touché par le "sombre », c'est-à-dire " assombri », couvert, voilé, mais encore au sentiment, c'est-à-dire à la tristesse, la pauvreté et au pessimisme. L' «ombre » n'est jamais que le côté obscur d'une chose ou la projection relativement à la lumière d'une silhouette sans profondeur.

Ce profilage syntaxique est à mettre en rapport avec le titre du poème "Chanson noire » qui fait partie d'un champ sémantique et thématique du « noir ». Ce qui nous intéresse ici c'est l'utilisation de ce champ du «noir » par le poète comme principe motivationnel de son chant / discours amoureux. Le titre d'ailleurs "Chanson noire» ne comporte pas d'article, il est déjà presque une incantation: Chanson noire.

\subsection{Parcours énonciatif de Chanson noire: motifs et thèmes}

Dans un premier temps: nous observons l'émergence d'une première instance s'énonçant sous une forme pronominale possessive, dans un paradigme du «noir » (1 $\left.1^{\text {ère}}\right)$, puis dans celui de l'évocation $\left(2^{\mathrm{e}}\right)$ avec "Mon doux mois / Ma songerie », " où vient rêvant, ma songerie aux murs de palmes »

A noter, il y a treize occurrences de la forme possessive du pronom à la première personne du singulier dans le poème. Ce qui dénote un rapport fort de dépendance et de possession chez le sujet ou plutôt peut-être pouvons-nous parler d'une instance énonciatrice "nonsujet » (en référence à la notion de J.C.Coquet Sujet / Non sujet) car celui qui énonce ce rapport de dépendance et de possession n'est alors pas dans le « je » d'une conscience énonciatrice.

On note une rupture d'isotopie à la $3^{\mathrm{e}}$ strophe et inchoativité du procès avec une thématisation du "double »: présence d'un couple oxymorique (faibles merveilles), couple thématique (faim / soif), 
couplet rythmique (collier collier) et enfin couple figural ( $\underline{\text { des soirs sans }}$ fin).

Sur le plan énonciatif, le chant d'amour s'auto-entretient dans la $3^{\text {e }}$ strophe avec une circularité textuelle associé au champ lexical et thématique que nous venons de voir et qui se poursuit à la $4^{\mathrm{e}}$ strophe avec le «tous les joncs».

La thématisation du «double » renforce l'idée de perte de soi, perte des repères tout en maintenant la présence de ce rapport très fort à la dépendance / possession "mes bras d'or, mes faibles merveilles ».Il y a également l'idée du double en soi, entre sujet et non sujet.

A la $4^{\mathrm{e}}$ strophe, il y a l'émergence de l'instance « je » qui amorce le rapport dialogique au «tu » ( sans t'avoir tressé / A ta fenêtre »)

Ce rapport dialogique au «tu » se confirme à la $5^{\mathrm{e}}$ strophe: « Dont tu m'atteins ».

Notons une rupture isotopique à la $6^{\mathrm{e}}$ strophe avec un paradigme du silence et de la perte avec « on jettera le manteau / Dessus ma face », on retrouve le voile devant la face qui impose le silence comme le voile du "sombre " en strophe 1. Le paradigme s'étend à la $7^{\mathrm{e}}$ strophe. L'instance non - sujet devient un " mon cri » intériorisé dans ce paradigme du silence. Le poète ne chante pas «je crie » mais bien «mon cri ». Il s'agit donc de cette instance non-sujet qui s'énonce. Autrement dit, le cri, distinct du «je », sort de la gorge dans la perte de tout contrôle. "Mon cri » est aussi métaphore de «ce langage perdu » qui est évoqué au début de la strophe. Le vers thématise sur la perte du langage poétique qui est aussi rappel du thème de la perte de l'objet d'amour que l'on retrouve notamment dans le chant courtois.

Remarquons le vers «ce trésor dans la fondrière » dont l'occurrence renvoie à une thématique sous-jacente au poème qui est celle de «l'œuvre au noir », c'est-à-dire la dynamique transformationnelle à partir du «noir » et qui rappelle le matériau chimique ou spirituel que l'on retrouve détaillé dans la littérature de l'alchimie, ou même dans une certaine littérature spirituelle ou poétique, notamment soufie, qui utilise le matériau du vécu amoureux douloureux comme point de départ pour une catalyse. Rappelons qu'Aragon place le recueil Elsa, dont est extrait ce poème, sous l'épigraphe du poète soufi Saadi.

Apparition d'un pronom "Elle » qui est nom propre à la $9^{\mathrm{e}}$ strophe "Pour Elle qu'un dernier saccage », il est énoncé avec une majuscule et thématise "l'amour du nom » des troubadours et du poète. « Nom - pronom » que l'on retrouve par ailleurs dans le théâtre d'Aragon.

À la $11^{\mathrm{e}}$, il y a phénomène de réflexivité forte avec l'utilisation du pronom réfléchi «moi-même » dans l'expression "aller au bout de moi-même ", notons une thématisation de l'identité, renforcé encore par la préposition « pour » qui véhicule un processus motivationnel qui 
est celui du « mouvement dans la profondeur ». La strophe s'achève sur le cri proférant le «je » déclaratif «je t’aime tant» ce qui marque un recouvrement des deux instances énonçantes de départ.

\subsection{Chanson noire exprime un rapport dialogique interne au} discours intérieur du poète

À partir de cette situation d'énonciation que nous venons d'évoquer, nous disons que la présence de la forme pronominale possessive à la première personne du singulier en particulier à la strophe 1 a une référence textuelle qui nous apparaît en lien avec une reprise anaphorique de " chanson noire » en titre. Ainsi, selon nous, le discours poétique « Mon sombre amour d'orange amère / Ma chanson d'écluse et de vent / Mon quartier d'ombre où vient rêvant / Mourir la mer ", etc., renvoie à Chanson noire et reprend l'incantation.

Autrement dit encore, une grande partie du discours poétique se confond avec l'expérience du poète marqué par le possessif: une expérience poétique et discursive qui n'est autre que Chanson noire et qui est constituée de rapports entre des formes perceptives, sémantiques et linguistiques dont les relations et faisceaux de relations marquent la sémantique du texte et conduisent à une sorte de rapport dialogique interne au discours intérieur du poète, ce que nous avons pu voir précédemment.

Tout d'abord, "chanson noire " est métaphore du langage poétique d'Aragon, mis en scène dans le discours amoureux du poème. Chanson noire est assimilée au "dit » ou au «chanté », elle est une chanson qui est un véritable objet: objet du discours de l'expérience amoureuse du poète dans lequel s'expriment plusieurs voix. Celle d'un «je» poétique, et celle d'une forme incantatoire, mélopée rythmée entre possession et dépossession, profilées sur le plan syntaxique (Mon sombre amour, mon quartier d'ombre...) et thématisée et thématisant les variations et motivations de sens du " noir» comme un principe d'absorption et de transformation ainsi que nous le retrouvons notamment dans les thématiques croisées du Chant d'amour du troubadour associé à la perte, l'Absence, l'œuvre au noir...

Enfin, penchons-nous sur l'allocutaire destinataire: le «tu ». Le «tu » renvoie-t-il à Elsa comme femme ou comme «nom secret»? Nous pensons au «senhal », sorte de nom fictif ou nom-écran que les troubadours donnaient à la Dame mais qui renvoyait davantage à la Joie sans motif ou Joie Pure, Joie absolue dans le cadre de la sublimation de l'objet d'amour comme forme absolue du Moi amoureux, ou encore signifiant primordial selon les thématiques psychanalytiques lacaniennes. Les thématiques développées autour du senhal sont: l'Absente, la Mort de la Dame, l'amour de loin, l'amour du nom... Et à ce titre ici c'est la présence du pronom personnel «Elle » qui apparaît comme un nom propre indéfini à la strophe 9 " Ah tandis encore qu'il bat / Ce cœur usé contre sa cage / Pour Elle ... » et qui renvoie à un principe féminin absolu, la Femme d'entre les Femmes. 


\section{Conclusion}

Dans notre travail, nous avons cherché à mettre en avant l'intérêt et la nécessité pour le professionnel de la traduction de développer une théorie du sens qui permette une lecture et interprétation sémantique comme préalable à toute traduction. Nous avons choisi de traiter un exemple d'analyse sémantique, celui du poème Chanson noire de L. Aragon afin d'illustrer nos propos théoriques.

L'analyse sémantique textuelle de Chanson noire a mis en évidence une reprise anaphorique permettant la création d'une sorte de mouvement polyphonique au sein du discours - qui est créé par le rapport du non-sujet à son expérience de possession / dépossession et son enchâssement sous la forme pronominale possessive dans le discours avec un « je » énonçant. Le « je » poétique pourrait s'adresser au chant d'amour ou "chanson noire » (présent dans le discours de possession narrative) entendu comme langage poétique quasi existentiel puisque le faire taire c'est une perte de la réalité poétique: ainsi énonce l’instance non-sujet «: et dressé trop peu de statues / À ta mémoire » (strophe 8). Le chant final apparaît comme un "crierdieu », qui est expression du «bout de moi-même »; cri qui ne fait qu'un avec son objet, l'objet du discours confondu avec son expression, le discours lui-même.

Pour la question de la traduction, si le traducteur n'est certainement pas, dans la perspective de ses choix traductifs, en nécessité de maîtriser le réseau de vecteurs transversaux et horizontaux qui charpentent une théorie des formes sémantiques, ni même d'être totalement familiarisé avec la sémantique textuelle, il n'en est pas moins invité par le présent article à découvrir certains éléments cruciaux de cette dernière. Nous lui avons ainsi présenté de nombreux points d'accroche d'une sémantique dont nous avons esquissé les caractéristiques essentielles. Il saura en tirer parti pour, nous l'espérons, aborder alors la complexité nouvelle du vouloir-dire et enrichir sa démarche de traducteur cibliste grâce à une prise en compte de facteurs qui, jusque là, ne lui étaient pas familiers.

\section{Annexe: Chanson noire}

Mon sombre amour d'orange amère

Ma chanson d'écluse et de vent

Mon quartier d'ombre où vient rêvant

Mourir la mer

Mon doux mois d'août dont le ciel pleut

Des étoiles sur les monts calmes

Ma songerie aux murs de palme

Où l'air est bleu 
Mes bras d'or mes faibles merveilles

Renaissent ma soif et ma faim

Collier collier des soirs sans fin

Où le cour veille

Dire que je puis disparaître

Sans t'avoir tressé tous les joncs

Dispersé l'essaim des pigeons

A ta fenêtre

Sans faire flèche du matin

Flèche du trouble et de la fleur

De l'eau fraîche et de la douleur

Dont tu m'atteins

Est-ce qu'on sait ce qui se passe

C'est peut-être bien ce tantôt

Que l'on jettera le manteau

Dessus ma face

Et tout ce langage perdu

Ce trésor dans la fondrière

Mon cri recouvert de prières

Mon champ vendu

Je ne regrette rien qu'avoir

La bouche pleine de mots tus

Et dressé trop peu de statues

À ta mémoire

Ah tandis encore qu'il bat

Ce cour usé contre sa cage

Pour Elle qu'un dernier saccage

La mette bas

Coupez ma gorge et les pivoines

Vite apportez mon vin mon sang

Pour lui plaire comme en passant

Font les avoines

Il me reste si peu de temps

Pour aller au bout de moi-même

Et pour crier Dieu que je t'aime

Je t'aime tant

\section{Références bibliographiques}

Aragon, Louis. « Chanson noire ». Elsa. Paris, Gallimard, 2010 [1959].

Cadiot, Pierre et Visetti, Yves-Marie. Pour une théorie des formes sémantiques: Motifs, profils, thèmes. Paris, 2001.

Cassirer, Ernst. Essai sur l'homme. Paris, Éditions de Minuit, 1975. 
Cixous, Hélène. «La venue à l'écriture ». Entre l'écriture. Paris, des femmes, 1985 .

Coseriu, Eugen. L'homme et son langage. Louvain-Paris, Peters, 2001.

Dupuis, France. Une approche linguistique et phénoménologique de la sémantique du mot amour. Université d'Orléans, Laboratoire ligérien de linguistique, thèse de doctorat, 2011.

Dupuis, France. "Approche phénoménologique de l'expression poétique de l'amour: le cas du mot hohoshiku ». In Julie Brock (dir.). Les tiges de mil et les pattes du héron. Lire et traduire les poésies orientales II. Paris: CNRS Editions, 2013: 279-285.

Dupuis, France. "Vers une sémantique linguistique et phénoménologique du mot amour ». In: Victor Rosenthal et Yves-Marie Visetti (éds.). Actes du colloque Programme perception sémiotique et socialité. "Le thème perceptif en linguistique ». Paris: CNRS Editions. (à paraître).

Dupuis, France et Lautel-Ribstein, Florence. "Motifs, profils et thèmes dans la traduction de Proust en anglais: une nouvelle méthodologie du traduire littéraire ». In Geneviève Henrot-Sostero, Florence Lautel-Ribstein, Magdalena Nowotna (dir.). Revue d'études proustiennes $n^{\circ} 1$ : Traduire A la recherche du temps perdu. Paris: Garnier (à paraître).

Jenny, Laurent. La parole singulière. Paris, Belin, 2009.

Lacan, Jacques. Ecrits. Paris, Seuil, 1966.

Ladmiral, Jean-René. Sourcier ou cibliste. Paris, Les Belles Lettres, 2014.

Ladmiral, Jean-René. "L'empire des sens ». In Marianne Lederer (dir.). Le sens en traduction. Paris-Caen: Minard-Lettres modernes. Collection Cahiers Champollion, 2006. 